\title{
Recovered or Resolved without Sequelae
}

National Cancer Institute

\section{Source}

National Cancer Institute. Recovered or Resolved without Sequelae. NCI Thesaurus.

Code C85257.

One of the possible results of an adverse event outcome where the subject recuperated and is free of any pathological conditions resulting from the prior disease or injury. 